\title{
Racial Disparities in the Utilization of Prophylactic Vaccinations and Inoculations in the U.S. Hospitals
}

\section{Mohammad A Faysel ${ }^{*}$ and Rachel T Assenza}

Medical Informatics Program, SUNY Downstate Medical Center, Brooklyn, NY, USA

"Corresponding author: Mohammad A Faysel, Medical Informatics Program, SUNY Downstate Medical Center, Brooklyn, NY, USA, Tel: 7182707693; Fax: 7182707739; Email: mohammad.faysel@downstate.edu

Received date: Sep 29, 2016; Accepted date: Oct 22, 2016; Published date: Oct 30, 2016

Copyright: ( 2016 Faysel MA, et al. This is an open-access article distributed under the terms of the Creative Commons Attribution License, which permits unrestricted use, distribution, and reproduction in any medium, provided the original author and source are credited.

\begin{abstract}
Objective: To examine racial disparities in the in-hospital utilization of frequently performed prophylactic vaccinations and inoculations in the U.S.

Methods: Using 2011 Nationwide Inpatient Sample (NIS) data, unadjusted Relative Risks (RRs) were calculated to compare the use of prophylactic vaccinations and inoculations between White and Black; White and Hispanic; White and Asian or Pacific Islander; and White and Native American for live birth; asthma; short gestation, low birth weight and fetal growth retardation; and other perinatal conditions. RRs were also calculated using patients' insurance statuses to further verify procedure use among new-borns.
\end{abstract}

Results: Whites were significantly less likely to receive prophylactic vaccinations and inoculations than all other races for live birth $(p<0.0001)$, and asthma $(p<0.05)$. Overall, White new-borns were also significantly less likely to receive prophylactic vaccinations than most of new-borns of other races when insurance status was compared.

Conclusion: Whites were less likely than other races to receive prophylactic vaccinations and inoculations in the U.S. hospitals.

Keywords: Vaccination disparities; Racial disparities; Prophylactic vaccination; Ethnic disparity; Health disparity

\section{Introduction}

Understanding racial disparities in the utilization of vaccination plays a vital role in the administration, production and supply management, and even development of new vaccines. Racial disparities in utilizing healthcare services have been well researched over the past several decades and the non-Black minority population has expanded dramatically over the same period of time. Numerous studies have documented persistent racial disparities in the use of vaccination in the U.S. and some of these studies have shown a decreasing trend of the racial disparities in the vaccination coverage [1-4]. Within the minority populations, these disparities impact all age groups and genders, and in addition, these disparities continue to exist regardless of patients' socioeconomic status [5,6]. Nationwide private and public sector efforts have been put to provide extended resources into researching causes of racial disparities in coverage of preventive vaccination and to eradicate the differences. However, effectiveness of these efforts is not always well documented and most of the published data on racial disparities in the utilization of vaccination do not include all the nonBlack minorities in the U.S. Prophylactic vaccinations and inoculations were the second most frequently performed medical procedures in the U.S. hospitals in 2011 [7]. This study examines racial disparities in the utilization of prophylactic vaccinations and inoculations across all hospitals in the U.S. by including all races and by using a more recent large nationwide dataset from the Healthcare Cost and Utilization Project's (H-CUP) Nationwide Inpatient Sample (NIS).
Eliminating racial and ethnic disparities within the healthcare system is fundamental in improving the overall quality of population health. The Institute of Medicine's 2001 report defined achieving equity as one of the six necessary elements for quality of care [8]. Furthermore; the U.S. should support delivery of healthcare services that do not vary due to one's gender, race, geographic location, and/or socioeconomic status [9].

Studies have evaluated socioeconomic factors as a potential contributor to racial disparities in the vaccination coverage across the U.S. In a study examining disparities in the Human Papilloma Virus (HPV) immunization, Niccolai et al. found adolescents who lived below the federal poverty threshold to be significantly less likely to complete vaccination compare to adolescents who had higher household incomes [5]. The study also found that after controlling covariates, Black and Hispanic adolescents were significantly less likely to complete vaccination than their White counterparts [5]. However, a study by Linn et al. indicates that although these socioeconomic issues undermine ideal vaccination coverage among minorities, they do not fully explain the lower vaccination percentages within minority racial and ethnic groups [6].

A study in 2014 found that racial/ethnic differences existed for the seven most widely distributed vaccines within the adult community, and higher vaccination coverage presented within the White population [10]. For example, in 2014, among older Hispanic adults, the pneumococcal immunization rate was as low as $40 \%$ vs. $74 \%$ for the non- Hispanic whites [11]. In the black community, among children aged 19-34 months, only $68 \%$ were fully vaccinated, compared with $78 \%$ of the non-Hispanic White children [12]. 
Page 2 of 6

However, Lu et al. showed that disparities in receiving vaccinations amongst children were significantly less than they had been in the past [13]. A study examining vaccination status of elderly American Indian and Alaskan Natives indicated that pneumococcal vaccination was at $58.1 \%$ for this minority versus $67.2 \%$ for their White counterparts [14]. A meta-analysis conducted by $\mathrm{Lu}$ et al. indicated that between 2007 and 2012, influenza vaccination amongst all adults increased significantly, however, racial and ethnic gaps in coverage persisted within that time period in most age groups [15].

Racial disparities within the healthcare system in general also exist regardless of patients' insurance status [9]. By including all the major races-Whites, Blacks, Hispanics, Asian or Pacific Islanders, and Native Americans; and all payers (insurance statuses), a study by Haque et al. examined a large dataset of over 3 million nationwide hospital discharges from NIS between 2001 and 2003, and showed persistent racial disparities in the in-hospital use of most of the top 10 frequently performed medical procedures in the U.S. hospitals [16]. The study also found Whites to be advantageous in receiving many of the procedures regardless of their insurance statuses [16].

Seasonal influenza was the eighth leading cause of death in 2009 which caused 53,692 deaths in the U.S [17]. Improved vaccination within the medically marginalized groups would likely reduce the need for ancillary medical care for which they are also underserved [17]. Study examining possible preventable hospitalization linked to influenza symptoms showed that Blacks had the highest rate and Hispanics had the second highest rate of such inpatient treatments [18].

Most of the published data focus on a single vaccination without including nationwide large data and they do not include all the nonBlack minorities when studying racial disparities. Most of them also do not include all the payer types (insurance statuses) which play a major role in receiving care in the hospitals. This study focuses on the overall use of prophylactic vaccinations and inoculations in the U.S. hospitals. All the non-Black minorities were included for comparison and all the payer types were also included for investigation of racial disparities in the utilization of prophylactic vaccinations and inoculations among new-borns.

\section{Methods}

\section{Data source and variable selection}

Nationwide Inpatient Sample (NIS) data, one of the most comprehensive hospital discharge summary data available, for the year 2011 were used for this study. NIS data were collected by Agency for Healthcare Research and Quality (AHRQ) which include all-payer inpatient-stay data from 1,049 hospitals across 46 states in the U.S. The dataset includes diagnosis and procedure codes in Clinical Classifications Software (CCS) generated codes. CCS codes combine related ICD-9-CM codes for diagnoses and procedures into more clinically meaningful categories [16]. For this study analysis, the second most frequently performed medical procedure in the U.S. hospitals in 2011, prophylactic vaccination and inoculation was used [7], and the top four principal diagnoses that are associated with this procedure were included [19]. These diagnoses are: live birth; asthma; short gestation, low birth weight and fetal growth retardation; and other perinatal conditions. The final dataset includes 729,257 records after excluding all missing values for race and age, and race coded as “Other". Patients' demographic information such as age, race, payer information (insurance status), principal diagnosis, and principal procedure were used in the data analysis.

\section{Study population}

All of the races-White, Black, Hispanic, Asian or Pacific Islander, and Native American; and all of the major payer categories-Medicaid, Medicare, and private insurance including Health Maintenance Organizations (HMO), self-payer uninsured, and no- charge uninsured were included in the study. The study population consisted of 54.7\% Whites, $16.3 \%$ Blacks, $23.2 \%$ Hispanics, 5\% Asians or Pacific Islanders and $0.8 \%$ Native Americans. Table 1 shows basic distribution of the study population by age group and race. Among all races, newborns constituted of $89.7 \%$ of the sample population. Within this age group (new-born), $55 \%$ were White, $14.8 \%$ were Black, $24.2 \%$ were Hispanic, $5.3 \%$ were Asian or Pacific Islander and $0.8 \%$ was Native American.

\begin{tabular}{|l|l|l|l|l|l|l|l|}
\hline Age group (in years) & New born & $\begin{array}{l}\text { Infant under } \\
\mathbf{1}(>1 \text { day) }\end{array}$ & Jan-17 & $\mathbf{1 8 - 4 4}$ & $\mathbf{4 5 - 6 4}$ & $\mathbf{6 5 - 1 0 2}$ & Total \\
\hline Race & $3,59,322$ & 4,173 & 5,973 & 6,389 & 11,687 & 11,586 & $3,99,130$ \\
\hline White & 96,963 & 1,299 & 5,742 & 4,432 & 7,522 & 3,104 & $1,19,062$ \\
\hline Black & $1,57,963$ & 1,455 & 3,397 & 1,756 & 2,463 & 2,054 & $1,69,088$ \\
\hline Hispanic & 34,865 & 266 & 358 & 152 & 255 & 501 & 36,397 \\
\hline Asian/Pacific Islander & 4,985 & 75 & 126 & 100 & 163 & 131 & 5,580 \\
\hline Native American & $6,54,098$ & 7,268 & 15,596 & 12,829 & 22,090 & 17,376 & $7,29,257$ \\
\hline Total & & & & & & & \\
\hline
\end{tabular}

Table 1: Study population by age group and race.

\section{Analysis of the study data}

The unit of the data analysis for this study is an individual discharge record. Unadjusted racial distribution of the study cohort was produced using summary statistics. In order to compare the procedure use among races, unadjusted Relative Risks (RRs) were calculated for White-Black, White-Hispanic, White-Asian or Pacific Islander, and White-Native American who were discharged for the following diagnoses: Live birth; asthma; short gestation, low birth weight and fetal growth retardation; or other perinatal conditions. Since 
Page 3 of 6

vaccination is a strongly recommended and common procedure for new-borns, calculation of unadjusted RRs were performed to further verify racial differences in the in-hospital use of prophylactic vaccinations and inoculations for live births using patients' payer categories. Missing payer information and payer type coded as "Other" was excluded for this calculation. The RRs were calculated and relevant 95\% Confidence Intervals (CI) and corresponding p-values were reported. When a cell value was less than five, the Fisher's Exact test was performed to calculate RR, its $95 \% \mathrm{CI}$ and related p-value. Base SAS version 9.4 (SAS Institute Inc.) was used for data analysis and result reporting.

\section{Results}

Table 2 shows the comparison of prophylactic vaccinations and inoculations use among White vs. all other races (Black, Hispanic, Asian or Pacific Islander, and Native American) for live birth; asthma; short gestation, low birth weight and fetal growth retardation; and other perinatal conditions in the U.S. hospitals.

\begin{tabular}{|c|c|c|c|c|}
\hline \multicolumn{5}{|l|}{ Relative Risks (RRs) } \\
\hline Diagnosis & $\begin{array}{l}\text { White-Black RR }(95 \% \mathrm{Cl}) \text {, } \\
\text { P value }\end{array}$ & $\begin{array}{l}\text { White-Hispanic RR }\left(\begin{array}{lll}95 \% & \mathrm{Cl}\end{array}\right), \mathrm{P} \\
\text { value }\end{array}$ & $\begin{array}{l}\text { White-Asian/Pacific Islander RR } \\
(95 \% \mathrm{Cl}), \mathrm{P} \text { value }\end{array}$ & $\begin{array}{l}\text { White-Native American } \\
\text { RR }(95 \% \mathrm{Cl}), \mathrm{P} \text { value }\end{array}$ \\
\hline Live Births & $0.72(0.72-0.73), p<0.0001$ & $0.73(0.73-0.74), p<0.0001$ & $0.81(0.80-0.82), p<0.0001$ & $0.85(0.82-0.88), p<0.0001$ \\
\hline Asthma & $0.78(0.67-0.92), p=0.003$ & $0.72(0.59-0.88), p=0.001$ & $0.64(0.40-1.01), p=0.053$ & $0.38(0.22-0.66), p<0.001$ \\
\hline $\begin{array}{l}\text { Short gestation, low } \\
\text { birth weight \& fetal } \\
\text { growth retardation }\end{array}$ & $0.7(0.52-0.95), p=0.024$ & $1.32(0.91-1.91), p=0.131$ & $1.22(0.58-2.56), p=0.585$ & $\mathrm{n} / \mathrm{a}$ \\
\hline $\begin{array}{l}\text { Other perinatal } \\
\text { conditions }\end{array}$ & $1.21(0.78-1.88), p=0.388$ & $1.4(0.91-2.16), p=0.121$ & $1.11(0.49-2.51), p=0.796$ & $0.5(0.19-1.31),{ }^{*} \mathrm{p}=0.143$ \\
\hline
\end{tabular}

*Fisher's Exact test was performed because minimum expected cell value was less than 5 . n/a: No RRs could be calculated because frequency of one or more cells was 0

Table 2: Relative Risks (RRs) of White vs. all other races.

\section{White vs. Black}

Whites were less likely to receive prophylactic vaccinations and inoculations in the hospitals for live birth; asthma; and short gestation, low birth weight and fetal growth retardation. Blacks were between $22 \%$ and $30 \%$ more likely to receive prophylactic vaccinations and inoculations for asthma $(\mathrm{p}<0.01)$, live births $(\mathrm{p}<0.0001)$, and gestation, low birth weight and fetal growth retardation $(\mathrm{p}<0.05)$ than Whites. There was no significant gap in the use of vaccinations and inoculations between White and Black for other perinatal conditions.

\section{White vs. Hispanic}

Whites were about $30 \%$ less likely to receive prophylactic vaccinations and inoculations than their Hispanic counterparts for asthma $(\mathrm{p}<0.0001)$, and live births $(\mathrm{p}<0.01)$. For short gestation, low birth weight and fetal growth retardation, and other perinatal conditions, Whites were between 1.3 and 1.4 times as likely to receive prophylactic vaccinations and inoculations as their Hispanic counterparts but these differences were not statistically insignificant.

\section{White vs. Asian or Pacific Islander}

Among live births, Whites were $20 \%$ less likely to receive prophylactic vaccinations and inoculations than Asians or Pacific Islanders $(\mathrm{p}<0.0001)$. However, there was no statistically significant gap between White and Black in vaccination usage for asthma; short gestation, low birth weight and fetal growth retardation; and other perinatal conditions.

\section{White vs. Native American}

Among live births, Whites were $15 \%$ less likely to receive prophylactic vaccinations and inoculations than their Native American counterparts $(\mathrm{p}<0.0001)$. For asthma, Whites were at least 1.6 times less likely to receive prophylactic vaccination and inoculation procedures than Native Americans $(\mathrm{p}<0.001)$. Among other perinatal conditions, there was no significant difference in the use of vaccination between White and Native American.

\section{Racial disparities by insurance status}

Table 3 shows the comparison of the utilization of prophylactic vaccinations and inoculations among new-borns (live birth) of all races compare to their White counterparts based on patients' insurance statuses (payer types). Among 654,098 live births, patients whose insurance statuses were missing $(1,330)$ or recorded as 'Other' $(17,666)$ were excluded for this analysis.

\section{Medicare}

Among Medicare recipients, new-born Whites were 57\% and 59\% more likely than new-born Blacks and new-born Hispanics, respectively, to receive vaccinations and inoculations $(\mathrm{p}<0.001)$ in the hospitals. There was no statistically significant difference between newborn Whites and new-born Asian or Pacific Islanders in the utilization of this procedure. 
Page 4 of 6

\begin{tabular}{|c|c|c|c|c|}
\hline \multicolumn{5}{|l|}{ Relative Risks (RRs) } \\
\hline Insurance Status & $\begin{array}{l}\text { White-Black RR }(95 \% \mathrm{Cl}), \mathrm{P} \text { - } \\
\text { value }\end{array}$ & $\begin{array}{l}\text { White- Hispanic RR } \\
(95 \% \text { Cl), P-value }\end{array}$ & $\begin{array}{l}\text { White-Asian or Pacific } \\
\text { Islander RR }(95 \% \text { Cl), P-value }\end{array}$ & $\begin{array}{l}\text { White-Native American RR }(95 \% \mathrm{Cl}), \mathrm{P} \text { - } \\
\text { value }\end{array}$ \\
\hline Medicare & $1.57(1.25-1.98), p<0.0001$ & $\begin{array}{l}1.59 \quad(1.08-2.34) \\
p=0.01\end{array}$ & $0.89(0.47-1.69), p=0.73$ & $\mathrm{n} / \mathrm{a}^{*}$ \\
\hline Medicaid & $0.72(0.72-0.73), p<0.0001$ & $\begin{array}{ll}0.7 & (0.69-0.70) \\
p<0.0001 & \end{array}$ & $0.71(0.70-0.73), P<0.0001$ & $0.98(0.93-1.03), p=0.38$ \\
\hline Private including HMO & $0.7(0.69-0.71), p<0.0001$ & $\begin{array}{ll}0.81 & (0.80-0.82) \\
p<0.0001 & \end{array}$ & $0.87(0.86-0.88), p<0.0001$ & $0.77(0.73-0.82), p<0.0001$ \\
\hline Uninsured (Self-pay) & $0.73(0.71-0.76), p<0.0001$ & $\begin{array}{ll}0.73 & (0.71-0.76) \\
p<0.0001 & \end{array}$ & $0.61(0.58-0.64), p<0.0001$ & $0.71(0.62-0.81), p<0.0001$ \\
\hline Uninsured (No charge) & $0.91(0.66-1.24), P=0.54$ & $\begin{array}{ll}0.48 & (0.40-0.56) \\
p<0.0001\end{array}$ & $1.2(0.74-1.96), P=0.45$ & $0.38(0.31-0.46), p<0.0001$ \\
\hline
\end{tabular}

Table 3: Relative Risks (RRs) comparing utilization of prophylactic vaccinations and inoculations among new-born of White race to that of all other races based on insurance status (payer type).

\section{Medicaid}

Among Medicaid recipients, new-born Whites were about 30\% less likely than new-borns of Black, Hispanic and Asian or Pacific Islander races in receiving vaccinations in the hospitals $(\mathrm{p}<0.001)$. There was no significant difference between new-born White and new-born Native American in receiving the vaccination and inoculation procedures.

\section{Private insurance}

Among the private insurance recipients including HMOs, new-born Whites were significantly less likely to receive prophylactic vaccinations and inoculations compare to their Black, Hispanic, Asian or Pacific Islander, and Native American counterparts. Among this insurance recipients, White new-borns were $30 \%$ less likely than Black new-borns $(\mathrm{p}<0.0001), 19 \%$ less likely than Hispanic new-borns $(\mathrm{p}<0001), 13 \%$ less likely than Asian or Pacific Islander new-borns $(\mathrm{p}<0.0001)$, and $23 \%$ less likely than Native American new-born's $(\mathrm{p}<0.0001)$ to receive this procedure.

\section{Self-pay (Uninsured)}

Among the self-payers who paid out of pocket for in-hospital services, White new-borns were significantly less advantageous in receiving prophylactic vaccinations and inoculations in the U.S. hospitals in 2011. Among the self-payers, the likelihoods of new-borns of Black, Hispanic, Asian or Pacific Islander, and Native American races receiving prophylactic vaccinations and inoculations was between 1.3 and 1.4 times more than new-borns of White race $(\mathrm{p}<0.0001)$.

\section{No-charge (Uninsured)}

Uninsured patients of this category were not charged for hospital services due to their financial inabilities. Among new-borns of this category, Whites were between 1.5 and 1.6 times less likely to receive vaccinations and inoculations compare to new-borns of Hispanic and Native American races respectively $(\mathrm{p}<0.0001)$.

\section{Discussion}

The strengths of this study are the using of a large nationwide inpatient data sample and inclusion of all the races in investigating racial disparities in the utilization of prophylactic vaccinations and inoculations in the U.S. hospitals. The study shows existence of disparities in the utilization of vaccinations across the U.S. hospitals.

Vaccinating new-born is highly recommended by health experts and sometimes required by some of the states in the U.S. Contrary to some previous study findings, the proposed study findings show that White new-borns were significantly less likely to receive prophylactic vaccinations and inoculations than all other races. This study further examines payers (insurance statuses) for all the races for live births to verify if insurance status plays any role in receiving prophylactic vaccinations and inoculations based on a new-borns race. In this comparison, White Medicare recipients were at least times more likely than Black or Hispanic Medicare recipients in receiving vaccination procedures. However, Whites who were self-paid uninsured or had private insurance were significantly less likely than all other races to receive vaccination procedures. New-borns of White Medicaid recipients were also less likely to receive these procedure compare to their Black, Hispanic, and Asian or Pacific Islander counterparts. Among the uninsured with no charge, White new-borns were at least 1.5 times less likely than Hispanic new-borns and Native American new-borns in receiving in-hospital vaccination procedures.

It is a well-researched fact that minorities have limited participation to preventive care in general, including vaccinations, as compared to their non-minority counterparts. Lu et al. showed that when adjusting for socioeconomic factors, racial and ethnic differences in obtaining vaccinations narrowed but was not eliminated [13]. Social class and demographic risk factors associated with minorities plagued by intense poverty also did not fully explain the racial and ethnic disparities [20]. Even Asian Americans with relatively high rates of income and health insurance often have low rates of preventative care [21]. There are factors, other than financial that impede minorities from obtaining equal vaccination status as compared to the non-Hispanic White majority in the U.S [13]. However, the proposed study findings show 
that White new-borns were less advantageous than other races in receiving vaccinations in the U.S. hospitals in 2011.

Among nationwide hospital stays for asthma, Whites were less likely to receive prophylactic vaccination and inoculation compare to their minority counterparts. The greatest disparity is evident when comparing Whites with Native Americans with asthma where Native Americans were at least 1.6 times as likely as Whites to receive this procedure. Whites were also significantly less likely than Blacks, Hispanics, and Asian or Pacific Islanders in receiving these procedures for asthma. Studies showed children with low socioeconomic status have fewer contacts with physicians and are less likely than their higher socioeconomic counterparts to receive vaccinations and are more frequently hospitalized for asthma. Black children are more likely to be hospitalized for asthma, suggesting less-than-optimum preventive care for this treatable condition [22]. Study showed Whites lead other races in socioeconomic status [9]. Contrary to these previous studies, the findings of this proposed study show that Whites were less advantageous than all other races in receiving in-hospital vaccinations for asthma.

Blacks were 1.3 times more likely than Whites in receiving prophylactic vaccinations and inoculations for short gestation, low birth weight and fetal growth retardation than Whites but there were no significant differences between Whites and other non-Black races in receiving vaccination for this condition. Studies have suggested that Black mothers are less likely to receive prenatal care than White mothers [22]. There are higher rates of low-birth weight babies and infant mortality within the Black population than within the White population. There is also evidence that the overall health of White children is superior to those children in the Asian and Hispanic communities [22]. However, the proposed study does not show statistically significant difference in using prophylactic vaccinations and inoculations for other perinatal conditions.

About $93 \%$ of the study population consisted of children who are under 18 years and $96 \%$ of them were new-borns. In 1994, a federally funded program, Vaccines for Children (VFC), was created to provide free vaccines to children who are uninsured, underinsured, Medicaid eligible and/or Native American or Alaskan Native [23]. Several studies showed measurable reductions in racial and ethnic disparities in vaccination rates for this population since the start of VFC $[23,24]$ but this study shows a wider gap in the utilization of vaccinations in the hospitals among new-borns where Whites were less advantageous than all of the other races in receiving these procedures. This study clearly shows racial disparities in the utilization of prophylactic vaccinations and inoculations across the U.S. hospitals despite various nationwide efforts to improve vaccination coverage. However, underlying factors causing these disparities cannot be determined from the study data. Numerous studies have attempted to identify the underlying causes of racial disparities [25-29]. Some of the major factors behind racial disparities in the utilization of vaccination related procedures could be parents' misconception of the adverse outcome of vaccination, education, socioeconomic status, access to healthcare services, language barrier, uneasiness toward serving the minority, minorities' mistrust toward the complex health systems, lack of availability of vaccines, [25] and "pervasive racial distrust" which causes negative stereotypes of physicians, resulting in resistance to adhere to prescribed medical protocol [26]. Individuals with multiple health concerns are more likely to obtain greater vaccine coverage overall [6]. Studies showed higher vaccination coverage presented within the White population as compared with most other groups
$[10,17]$ However, this study mostly contradicts with the findings of some previous studies. With population trends pointing towards a larger percentage of the overall U.S. population being represented by various minority groups, it is imperative that minority participation in obtaining preventive vaccinations be optimized. This would positively impact public wellness and healthcare costs.

\section{Conclusion}

This study uses a large volume of more recent nationwide inpatient data and indicates that minorities are utilizing prophylactic vaccinations and inoculations in the U.S. hospitals to a greater degree than past research indicated. Future research should include large data from different sources to further investigate these findings. Although health-related disparity elimination has been a focus of national attention, measurable racial disparities continue to exist. While differences have narrowed as compared to some earlier studies, this study shows that disparities still exists in the utilization of vaccinations. Examining patients' socioeconomic status or other patient details to further identify the primary causes of racial disparity is beyond the focus of this study. Future research should identify and focus on the combination of personal, social, culturally based and environmental factors in eradicating racial disparities in healthcare.

\section{References}

1. Yoo BK, Kasajima M, Phelps CE, Fiscella K, Bennett NM, et al. (2011) Influenza vaccine supply and racial/ethnic disparities in vaccination among the elderly. Am J Prev Med 40: 1-10.

2. Morita JY, Ramirez E, Trick WE (2008) Effect of a school-entry vaccination requirement on racial and ethnic disparities in hepatitis $\mathrm{B}$ immunization coverage levels among public school students. Pediatrics 121: e547-e552.

3. Walker AT, Smith PJ, Kolasa M, Centers for Disease Control and Prevention (CDC) (2014) Reduction of racial/ethnic disparities in vaccination coverage 1995-2011. MMWR 63: 7-12.

4. Flores G, Olson L, Tomany-Korman SC (2005) Racial and ethnic disparities in early childhood health and health care. Pediatrics 115: e183-193.

5. Niccolai LM, Mehta NR, Hadler JL (2011) Racial/Ethnic and poverty disparities in human papillomavirus vaccination completion. Am J Prev Med 41: 428-433.

6. Linn ST, Guralnik JM, Patel KV (2010) Disparities in influenza vaccine coverage in the United States, 2008. J Am Geriatr Soc 58: 1333-1340.

7. Pfuntner A, Wier LM, Stocks C (2011) Most frequent procedures performed in U.S. hospitals, 2011. Healthcare Cost and Utilization Project (HCUP) Statistical Briefs. Rockville (MD): Agency for Healthcare Research and Quality (US).

8. Leavitt M (2001) Medscape's response to the Institute of Medicine Report: Crossing the quality chasm: a new health system for the 21 st century. Med Gen Med 3: 2.

9. Trivedi AN, Zaslavsky AM, Schneider EC, Ayanian JZ (2006) Relationship between quality of care and racial disparities in Medicare health plans. JAMA 296: 1998-2004.

10. Williams WW, Lu PJ, O'Halloran A, Kim DK, Grohskopf LA, et al. (2016) Surveillance of Vaccination Coverage Among Adult Populations - United States, 2014. MMWR Surveill Summ 65: 1-36.

11. Pfizer Inc (2014) Vaccination in the Hispanic community: a cause for concern and a call to action.

12. Centers for Disease Control and Prevention (CDC) (2005) Health disparities experienced by black or African Americans-United States. JAMA 293: 922-923. 
Citation: Faysel MA, Assenza RT (2016) Racial Disparities in the Utilization of Prophylactic Vaccinations and Inoculations in the U.S. Hospitals. J Health Med Inform 7: 245. doi:10.4172/2157-7420.1000245

Page 6 of 6

13. Lu PJ, O'Halloran A, Williams WW, Lindley MC, Farrall S, et al. (2015) Racial and Ethnic Disparities in Vaccination Coverage Among Adult Populations in the U.S. Am J Prev Med 49: S412-S425.

14. Lindley MC, Groom AV, Wortley PM, Euler GL (2008) Status of influenza and pneumococcal vaccination among older American Indians and Alaska Natives. Am J Public Health 98: 932-938.

15. Lu PJ, O'Halloran A, Bryan L, Kennedy ED, Ding H, et al. (2014) Trends in racial/ethnic disparities in influenza vaccination coverage among adults during the 2007-08 through 2011-12 seasons. Am J Infect Control 42: 763-769.

16. Haque SS, Faysel MA, Khan HM (2010) Racial differences in the use of most commonly performed medical procedures in the United States. J Health Dispar Res Pract 4: 14-25.

17. Wang J, Munshi KD, Hong SH (2014) Racial and ethnic disparities in influenza vaccinations among community pharmacy patients and noncommunity pharmacy respondents. Res Social Adm Pharm 10: 126-140.

18. Russo CA, Andrews RM, Coffey RM (2003) Racial and ethnic disparities in potentially preventable hospitalizations, 2003. Healthcare Cost and Utilization Project (HCUP) Statistical Briefs. Rockville (MD): Agency for Healthcare Research and Quality (US).

19. Elixhauser A, Steiner CA (1996) Most common diagnoses and procedures in U.S. community hospitals, 1996. Summary, HCUP Research Note. Agency for Health Care Policy and Research, Rockville.

20. Tsui J, Gee GC, Rodriguez H (2013) Exploring the role of neighborhood socio-demographic factors on HPV vaccine initiation among lowincome, ethnic minority girls. J Immigr Minor Health 15: 732-740.
21. American College of Physicians (2010) Racial and ethnic disparities in health care.

22. Chen E, Martin AD, Matthews KA (2006) Understanding health disparities: the role of race and socioeconomic status in children's health. Am J Public Health 96: 702-708.

23. Centers for Disease Control and Prevention (CDC) (2014) Vaccines For Children (VFC) Program.

24. Weil AR (2016) Vaccine Discovery, Production, And Delivery. Health Aff (Millwood) 35: 187.

25. Soyemi K, Medina-Marino A, Sinkowitz-Cochran R, Schneider A, Njai R, et al. (2014) Disparities among 2009 pandemic influenza A (H1N1) hospital admissions: a mixed methods analysis-Illinois. PLoS ONE 9: e84380.

26. Dovidio JF, Penner LA, Albrecht TL, Norton WE, Gaertner SL, et al. (2008) Disparities and distrust: the implications of psychological processes for understanding racial disparities in health and health care. Soc Sci Med 67: 478-486.

27. Mendoza FS (2009) Health disparities and children in immigrant families: a research agenda. Pediatrics 3: S187-S195.

28. Ahluwalia IB, Ding H, Harrison L, D'Angelo D, Singleton JA, et al. (2014) Disparities in influenza vaccination coverage among women with liveborn infants: PRAMS surveillance during the 2009-2010 influenza season. Public Health Rep 129: 408-416.

29. Cooper LA, Hill MN, Powe NR (2002) Designing and evaluating interventions to eliminate racial and ethnic disparities in health care. J Gen Intern Med 17: 477-486. 